\title{
Avaliação do modelo reduzido CAARC: abordagem experimental, analítica e numérica
}

\section{Evaluation of the reduced CAARC model: experimental, analytical and numerical approach}

\author{
Patrick de Oliveira Batista da Costa ${ }^{1 *}$, Danila dos Anjos Ribeiro ${ }^{2}$
}

\begin{abstract}
RESUMO
As estruturas estão gradualmente mais sensíveis a efeitos dinâmicos (e.g. ventos, sismos) devido a demanda por construções esbeltas constituídas de materiais mais leves e resistentes. Assim, para um melhor entendimento do comportamento das estruturas é relevante que se realize uma análise teórica-analítica, numérica e experimental em conjunto, alcançando um resultado mais apurado. Diante disso, buscou-se, neste estudo realizar estas três análises através da construção de um modelo reduzido de um edifício alto padrão, i.e., CAARC, respeitando as leis de semelhança. Os cálculos do modelo experimental assim como a análise modal do modelo analítico com a consideração de incertezas foram implementados em uma rotina computacional na linguagem Python 3. O modelo numérico foi desenvolvido no software de elementos finitos Abaqus ${ }^{\circledR}$. Em síntese, com a análise experimental do modelo reduzido foi capaz de encontrar a escala de velocidades para um possível ensaio em túnel de vento. Os modelos analítico e numérico apresentaram resultados próximos, porém distintos do modelo experimental.
\end{abstract}

Palavras-chave: Análise modal; Modelo reduzido; CAARC.

\begin{abstract}
Structures are gradually more sensitive to dynamic effects (e.g. wind, earthquakes) due to the demand for slender buildings made of lighter and stronger materials. Thus, for a better understanding of the behavior of structures it is relevant to perform a joint theoretical-analytical, numerical and experimental analysis, achieving a more accurate result. Therefore, this study sought to perform these three analyses through the construction of a reduced model of a high-standard building, i.e., CAARC, respecting the similarity laws. The calculations of the experimental model as well as the modal analysis of the analytical model with the consideration of uncertainties were implemented in a computational routine in Python 3 language. The numerical model was developed in the finite element software Abaqus®. In summary, with the experimental analysis of the reduced model it was possible to find the velocity range for a possible wind tunnel test. The analytical and numerical models presented close results, but different from the experimental model.
\end{abstract}

Keywords: Modal analysis; Reduced model; CAARC.

${ }^{1}$ UFRGS - Universidade Federal do Rio Grande do Sul. *E-mail: patrick.costa@ufgrs.br

${ }^{2}$ USP - Universidade de São Paulo 


\section{INTRODUÇÃO}

A busca por construções com estruturas cada vez mais esbeltas tem como um dos fatores decorrentes o crescimento populacional exacerbado, e, somado a tecnologia dos materiais de maneira a obter elementos mais leves e otimizados visando menor custo, torna-se essas estruturas cada vez mais sensíveis a demandas dinâmicas podendo ser levadas a ruína, e.g., vento, sismos, equipamentos em funcionamento, deslocamentos de pessoas, movimentação de veículos, entre outras (ROSSATO, 2017).

Segundo Rossato (2020), mesmo que as ações dinâmicas não atinjam o estado limite último (ELU) da estrutura, as vibrações por exemplo, podem causar certo desconforto aos usuários, de modo a não respeitar o estado limite de serviço (ELS).

A utilização da análise numérica, através do Método dos Elementos Finitos (MEF) por exemplo, surge como uma alternativa para buscar uma melhor precisão do desempenho das estruturas submetidas a cargas estáticas e dinâmicas, tal como o comportamento dos materiais com modelos lineares e não lineares, próximos da estrutura real. São muito utilizados para realizar análises de estruturas complexas e/ou submetidas a carregamentos que culminam em deslocamentos e deformações grandes para um posterior dimensionamento mais preciso, seguro e econômico, dada a resposta da estrutura.

Destarte, de acordo com Beck (2019) e Rocha (2021), os modelos matemáticos, especificamente os utilizados na análise de engenharia podem conter várias fontes de incertezas/erros, e.g., incertezas fenomenológicas, erro estatístico, erro humano, erro de modelo, e variabilidade intrínseca de quantidades físicas. Sendo assim, medições experimentais têm mostrado que as propriedades dinâmicas da estrutura não são iguais ou mesmas próximas daquelas calculadas em projeto. Outrossim, realizar análises considerando tais incertezas, i.e., projetar uma estrutura através de uma abordagem estocástica pode resultar em um custo computacional muito elevado.

O motivo e a preferência em se realizar uma análise experimental foi descrito acima, porém este tipo de análise pode-se exigir um alto valor financeiro. Então, como meio de contornar esta questão, opta-se por análises experimentais com modelos reduzidos, de maneira a respeitar as leis de semelhança.

Outro fator que endossa a utilização da análise experimental se deve a dificuldade de avaliar a resposta de certas estruturas sob carregamento dinâmico, por exemplo. A NBR 6123 (ABNT, 1988) e sua atual revisão preconiza para estruturas com altura acima 
de 150 metros, formas que não há na norma e/ou em situações de vizinhança, ensaios em túnel de vento. Estes ensaios em túnel de vento utilizam modelos experimentais em escala reduzida.

Nesse contexto, o trabalho tem por objetivo realizar uma análise modal experimental de um modelo reduzido a ser construído de forma a possibilitar o ensaio em túnel de vento. Ainda, comparar o modelo experimental com o modelo analítico levando em consideração as incertezas, e com o modelo numérico, através da ferramenta computacional ABAQUS®.

\section{MODELO EXPERIMENTAL}

\section{A escolha do protótipo}

Na Austrália, em 1969, com a finalidade de realizar a comparação de ensaios de simulação do vento natural em túneis de vento, Wardlaw e Moss criaram a especificação de um edifício alto padronizado (MELBOURNE, 1980).

Até 1975 , cinco centros de pesquisa já haviam realizado medições no modelo "CAARC Standard Tall Building."

Atualmente, o CAARC já possui um amplo banco de dados em estudo com diversas finalidades, utilizando diversos mecanismos de ensaio. No Brasil também já vem apresentando diversos estudos realizados sobre: tais como as produções de Oliveira (2009), Sartori (2010), Vanin (2011), por exemplo.

As propriedades deste edifício alto, i.e., o CAARC pode ser verificado na Tabela 1 e na Figura 1, respectivamente.

Tabela 1 - Propriedades do CAARC

\begin{tabular}{cccc}
\hline Dimensões [m] & $\begin{array}{c}\text { Frequência } \\
\text { fundamental }[\mathrm{Hz}]\end{array}$ & $\begin{array}{c}\text { Massa específica } \\
{\left[\mathrm{kg} / \mathrm{m}^{3}\right]}\end{array}$ & $\begin{array}{c}\text { Amortecimento } \\
{[\%]}\end{array}$ \\
\hline $30.48 \times 45.72 \times 182.88$ & $0.2^{*}$ & 160 & 1.0 \\
\hline
\end{tabular}

* mesma frequência na direção plana X e Y.

Fonte: Os Autores (2022) 
Figura 1 - Dimensões do CAARC

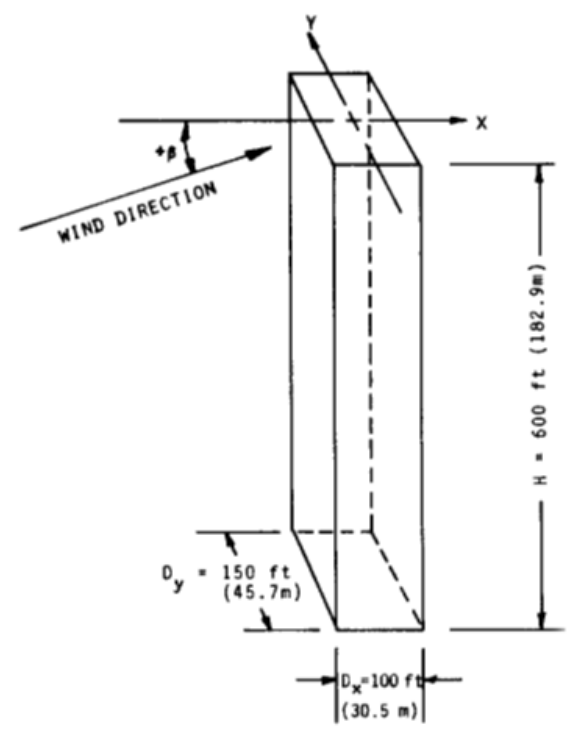

Fonte: Melbourne (1980)

\section{Modelo reduzido}

Conforme mencionado anteriormente, o ensaio em modelo reduzido se deve ao fato de redução de tempo de construção e principalmente de custo. Não obstante, para que o modelo reduzido seja capaz de reproduzir o mesmo comportamento do protótipo para uma certa análise, as leis de semelhança devem estar de acordo (BALENDRA, 1993).

Sendo assim, como o presente estudo apresenta como finalidade o ensaio do modelo reduzido ser realizado em um possível túnel de vento, a construção desse modelo assim como as leis de semelhança escolhidas será específica para este estudo.

O ensaio em túnel de vento de camada limite de ciclo aberto ou fechado admitem dois tipos de testes. O primeiro tipo de teste é denomindado estático, i.e, são confeccionados modelos reduzidos rígidos que são instrumentados com tomadas de pressão onde são medidas instantaneamente as pressões devidas à ação do vento, devendo, portanto, apenas observar a escala geométrica do modelo com a escala do vento. Já o segundo teste é chamado de dinâmico pois utiliza-se um modelo aeroelástico, i.e., que representa o comportamento dinâmico da estrutura, e pode ser classificado em três classes distintas: réplicas, modelos seccionais e modelos equivalentes. As réplicas geralmente são mais custosas pois resulta em uma reprodução completa em escala das características dinâmicas do protótipo. Já os modelos seccionais são interessantes quando é possível considerar o vento como escoamento bidimensional, e.g., pontes, chaminés, 
torres, entre outros. Por fim, a terceira classe, escolhida para o presente estudo, é denominada de modelos equivalentes, pois este modelo trata-se de alguma analogia mecânica de forma a representar as características dinâmicas do protótipo, i.e., CAARC.

\section{Modelo equivalente}

De acordo com Carneiro (1993) através da análise dimensional é possível estabelecer as leis de semelhança para um determinado modelo reduzido, i.e., a partir de três grandezas de base obtém-se as grandezas derivadas. Das grandezas derivadas resultantes, a de maior interesse neste estudo é a escala de velocidade básica do vento que deverá ser simulada em um túnel de vento.

No que diz respeito as grandezas de base, foram escolhidas a escala de comprimento $\left(\lambda_{L}\right)$, escala de massa específica $\left(\lambda_{\rho}\right)$, e escala de frequência $\left(\lambda_{f}\right)$. A escala de comprimento foi determinada de modo a possibilitar a construção do modelo e obedecer a disposição do mesmo dentro do túnel de vento. A escala de massa específica foi atribuída igual a do protótipo, e a escala de frequência será obtida somente após a medição da frequência do modelo reduzido equivalente já construído. A Equação 1 e 2 representa as escalas de comprimento e de massa específica, respectivamente.

$$
\begin{aligned}
& \lambda_{L}=\frac{L_{m}}{L_{p}} \rightarrow \frac{1}{406.4} \\
& \lambda_{\rho}=\frac{\rho_{m}}{\rho_{p}} \rightarrow \frac{160}{160} \rightarrow \frac{1}{1}
\end{aligned}
$$

Em que $L_{m}$ é o comprimento do modelo reduzido; $L_{p}$ o comprimento do protótipo; $\rho_{m}$ massa específica do modelo reduzido; $\rho_{p}$ massa específica do protótipo.

Dado as escalas de comprimento e massa específica, a Tabela 2 mostra o modelo reduzido equivalente já na escala a ser construída.

Tabela 2 - Escala do modelo reduzido equivalente - CAARC

\begin{tabular}{cccc}
\hline Altura [m] & Largura - dir. X [m] & Comprimento - dir. Y [m] & Massa total [kg] \\
\hline 0.45 & 0.075 & 0.1125 & 0.6075 \\
\hline
\end{tabular}

Fonte: Os Autores (2022) 


\section{Projeto e construção}

A fim de representar de maneira mais precisa as dimensões e a massa total do modelo reduzido equivalente, optou-se por dividir o edifício em 5 pavimentos de mesmo pé direito, onde cada pavimento deverá obter uma massa correspondente a um quinto do valor da massa total do CAARC, i.e., 121,5 gramas por pavimento. Tendo em vista que o critério de escolha dos materiais foram a leveza, a flexibilidade de forma a evitar o dano localizado, a Tabela 3 expõe os materiais escolhidos a serem utilizados, suas quantidades e respectivas massas. A Figura 2 apresenta o projeto do modelo reduzido.

Tabela 3 - Propriedades dos materiais utilizados

\begin{tabular}{ccccc}
\hline Materiais & Quant. & $\begin{array}{c}\text { Dimensões } \\
{[\mathrm{mm}]}\end{array}$ & $\begin{array}{c}\text { Massa / unidade } \\
{[\mathrm{kg}]}\end{array}$ & $\begin{array}{c}\text { Massa total } \\
{[\mathrm{kg}]}\end{array}$ \\
\hline $\begin{array}{c}\text { Placas de } \\
\text { acrílico }\end{array}$ & 5 & $112.5 \times 75 \times 3$ & 0.031 & 0.155 \\
\hline $\begin{array}{c}\text { Fios de arame } \\
\text { galvanizado }\end{array}$ & 4 & $\Phi 2.1$ & 0.013 & 0.052 \\
\hline $\begin{array}{c}\text { Chumbada de } \\
\text { pesca }\end{array}$ & 40 & Comp. 450 & 0.010 & 0.4 \\
\hline$\Sigma$ & - & $\Phi 12$ & - & 0.607 \\
\hline
\end{tabular}

Fonte: Os Autores (2022)

Cabe ressaltar que para a balança utilizada ajustada para o nível de precisão em gramas, não houve variação da massa medida entre os elementos, podendo considerar o desvio padrão para cada material igual zero.

A montagem da estrutura foi desenvolvida através da fixação/engaste dos quatro fios de arame galvanizado em uma base de placa MDF (47 x 32 × $2 \mathrm{~cm})$, através da cola Tek Bond. Essa mesma cola foi utilizada para a ligação entre os fios de arame com a placa de acrílico, de maneira a tentar reproduzir um comportamento monolítico entre a laje/viga com os pilares.

O complemento da massa de cada pavimento, representado pelas chumbadas de pesca, foi posicionado a fim de manter a simetria geométrica necessária para conservar a frequência fundamental nas duas direções, conforme o protótipo. Além disso, foram colados em pares na placa de acrílico, totalizando 8 chumbadas por piso. 
Figura 2 - Projeto CAARC: (a) Planta baixa (b) Elevação (c) Isométrico (3D)

a)

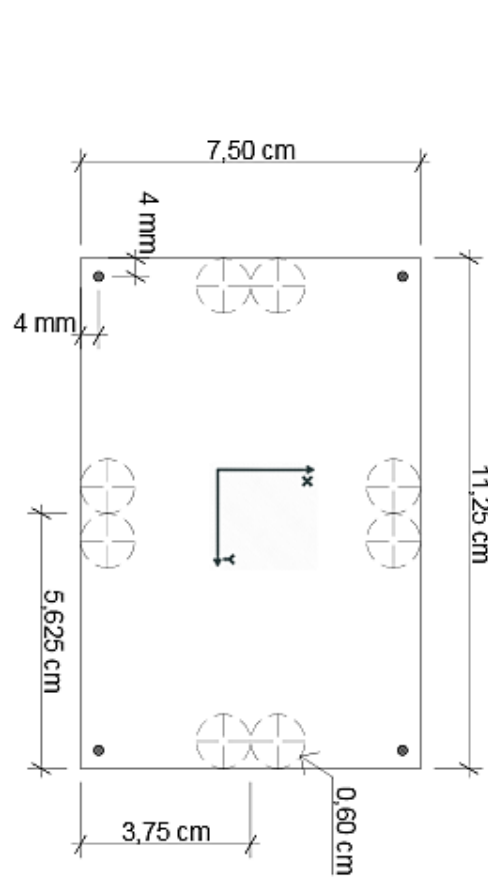

b)

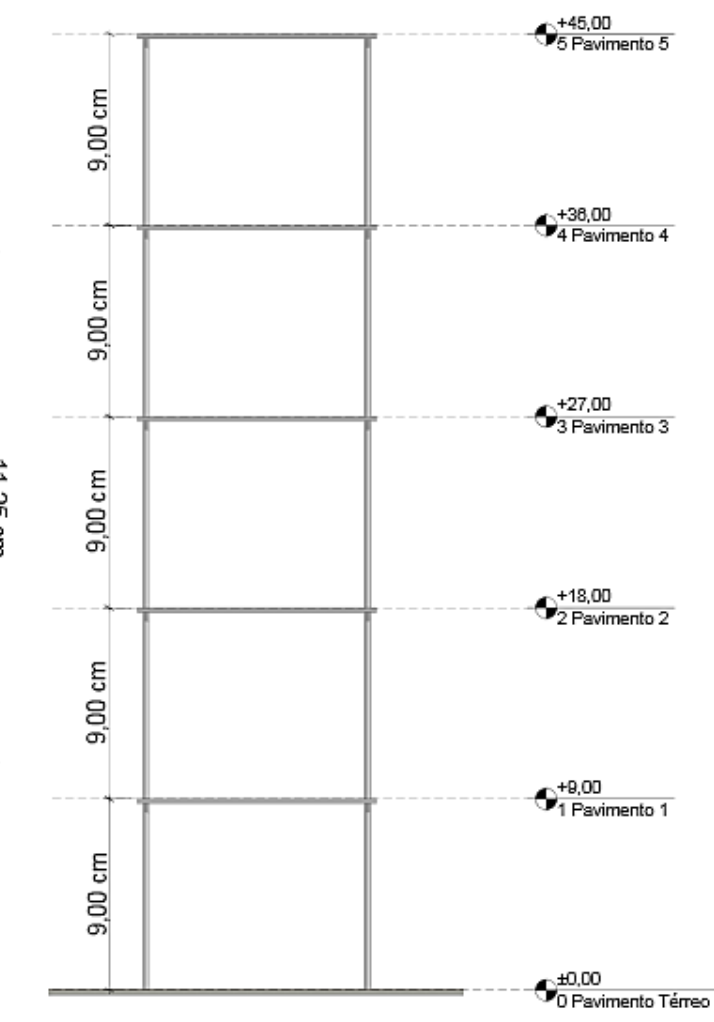

c)

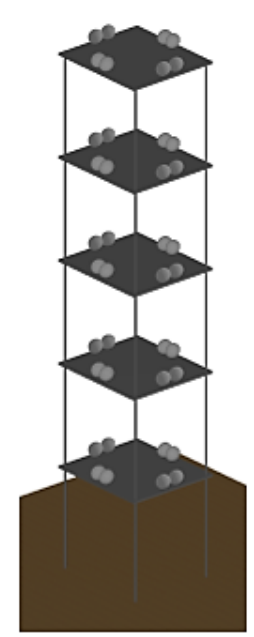

Fonte: Os autores (2022)

\section{Instrumentação}

Com o propósito de descobrir a escala de frequência modelo reduzido, a estrutura foi instrumentada com um acelerômetro triaxial MPU-6050, posicionado no centroide do último pavimento. Para a instalação desse dispositivo, empregou-se a fita dupla-face fina de modo a evitar possíveis ruídos na leitura devido ao amortecimento causado entre o dispositivo e a placa de acrílico, após uma condição inicial de deslocamento.

Para leitura e aquisição dos dados utilizou-se o microcontrolador Arduino UNO integrado ao acelerômetro através do barramento de comunicação serial sob protocolo $\mathrm{I}^{2} \mathrm{C}$. Este protocolo utiliza como ligação as portas de entrada analógica A4 e A5 do Arduino ligados no acelerômetro nas entradas SDA (Serial Data) e SCL (Serial Clock), respectivamente. A fonte alimentação utilizada foi de $5 \mathrm{~V}$.

A Figura 3 apresenta a ligação realizada através do Arduino UNO e o acelerômetro triaxial MPU-6050, bem como a protoboard utilizada como suporte para o microcontrolador. 
Figura 3 - Ligação Arduino UNO e acelerômetro MPU-6050

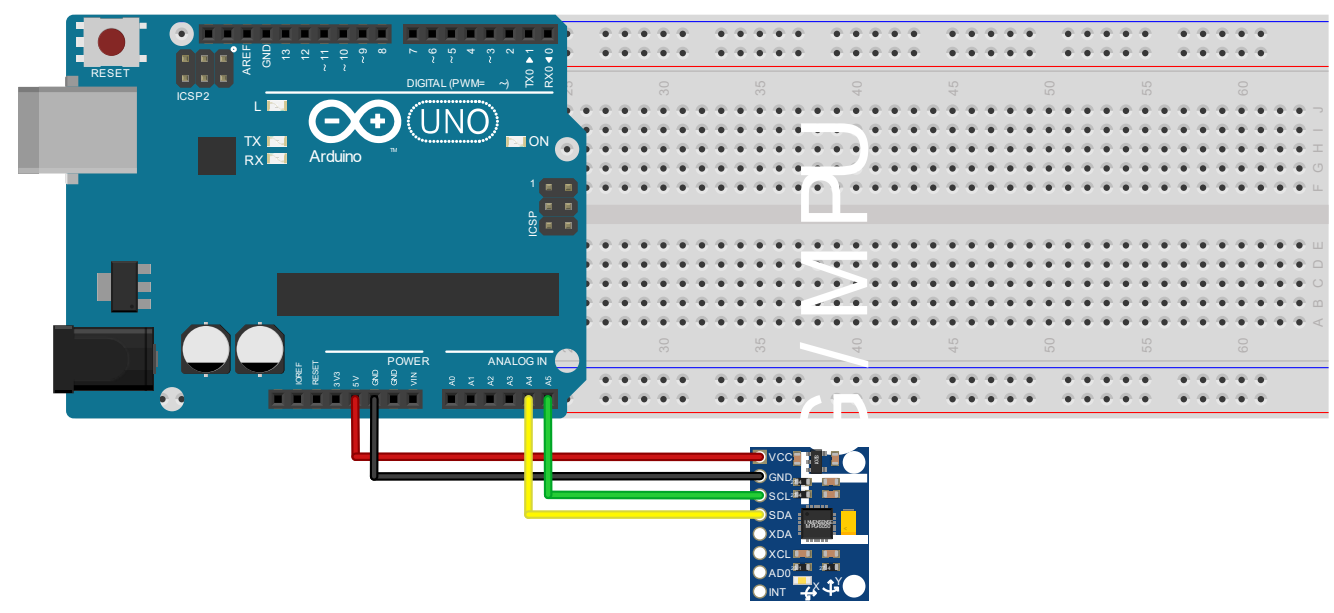

Fonte: Os autores (2022)

As linguagens de programação utilizadas para a realização da instrumentação e medição dos sinais foram C++ (Arduino UNO) e Python 3 (Jupter Notebook) e o código programado encontra-se disponível no GitHub através do endereço de acesso: https://github.com/costapatrick/CAARC-Equivalent-Reduced-Model, com o nome dos arquivos: ReadMPU6050.ino e read_Serial.ipynb. O modelo reduzido contruído e instrumentado é apresentado através da Figura 4 a seguir.

Figura 4 - Modelo Reduzido construído e instrumentado

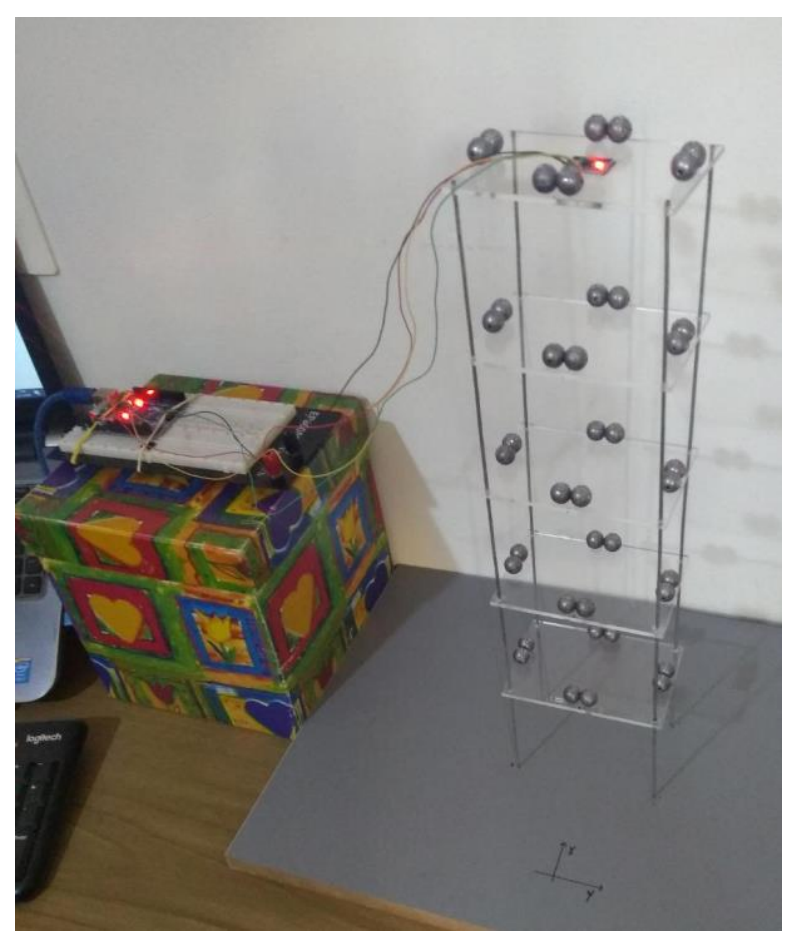

Fonte: Os autores (2022) 


\section{Leitura dos sinais}

Para a detecção da frequência fundamental nas duas direções (X e Y no plano) será medido o sinal da resposta da estrutura em forma de aceleração, dado uma condição inicial de deslocamento na estrutura.

A taxa de comunicação utilizada foi de 115.200 bits por segundo, e o tempo de leitura foi ajustado para 10 segundos, dada a taxa média de amostragem de $367.9 \mathrm{~Hz}$.

$\mathrm{O}$ acelerômetro foi calibrado e programado para medição das acelerações em X e Y no plano. Ao todo foram realizadas 6 medições, 3 na direção X (Figura 5, 6 e 7) e 3 na direção Y (Figura 8, 9 e 10). A fim de uma melhor aproximação e suavização do sinal, utilizou-se o método fitdecay presente na biblioteca MRPy, no qual se realiza uma interpolação entre as curvas de maneira a evitar os ruídos causados pela medição real.

Figura 5 - Medição 1: aceleração na direção X

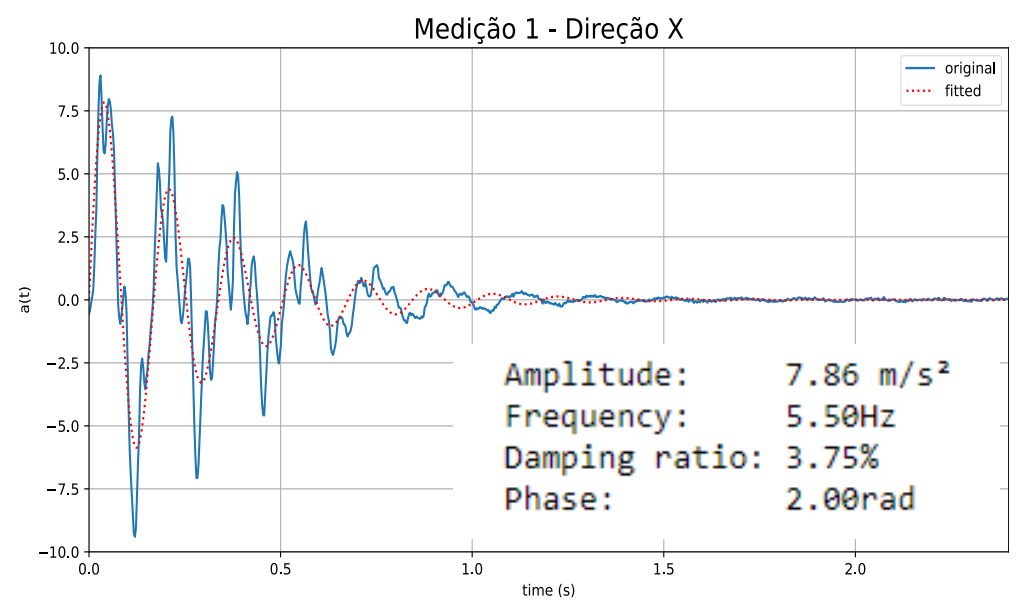

Fonte: Os autores (2022)

Figura 6 - Medição 2: aceleração na direção X

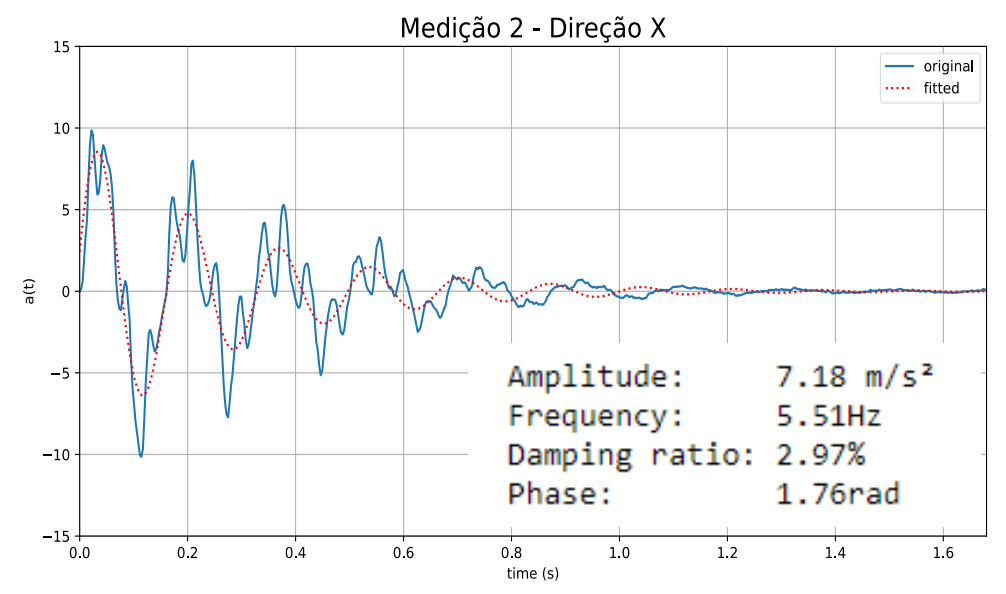

Fonte: Os autores (2022) 
Figura 7 - Medição 3: aceleração na direção X

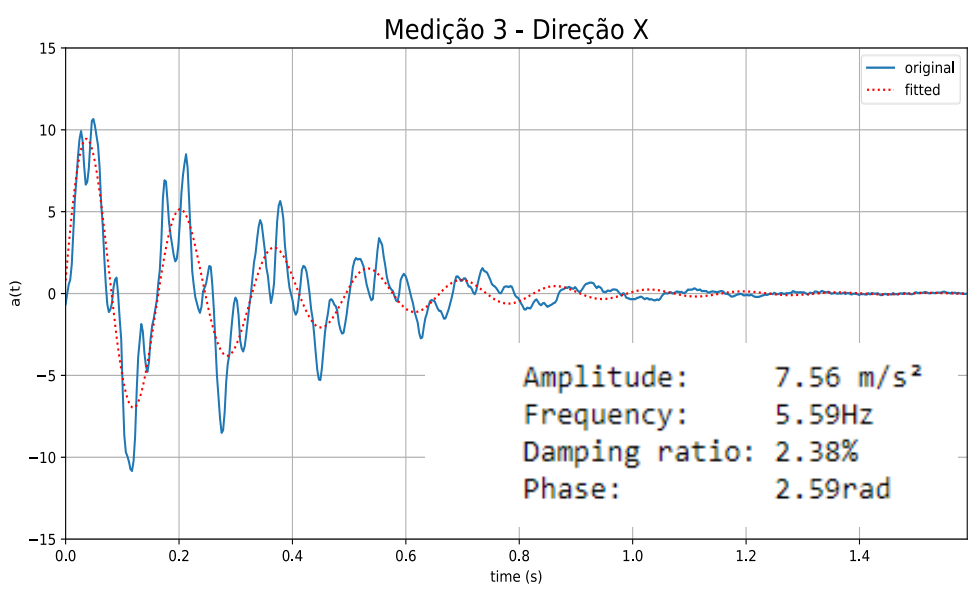

Fonte: Os autores (2022)

Figura 8 - Medição 1: aceleração na direção Y

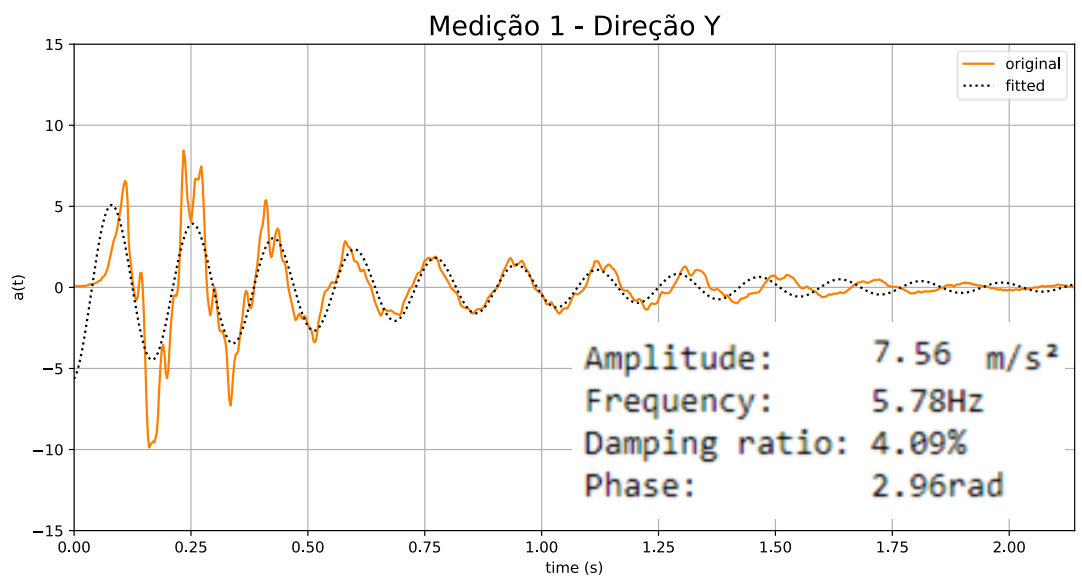

Fonte: Os autores (2022)

Figura 9 - Medição 2: aceleração na direção Y

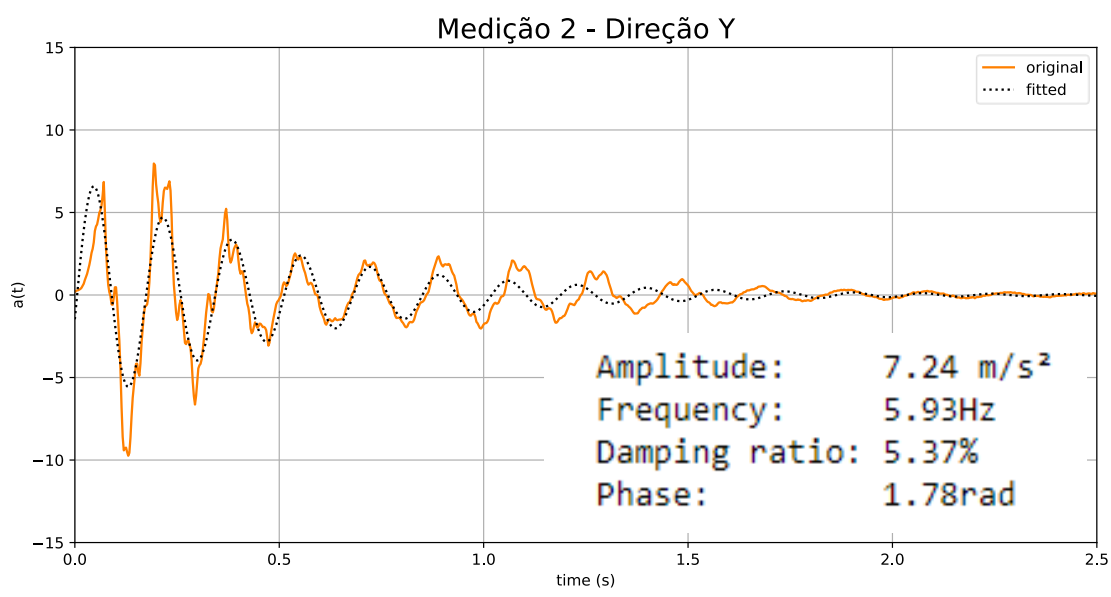

Fonte: Os autores (2022) 
Figura 10 - Medição 3: aceleração na direção Y

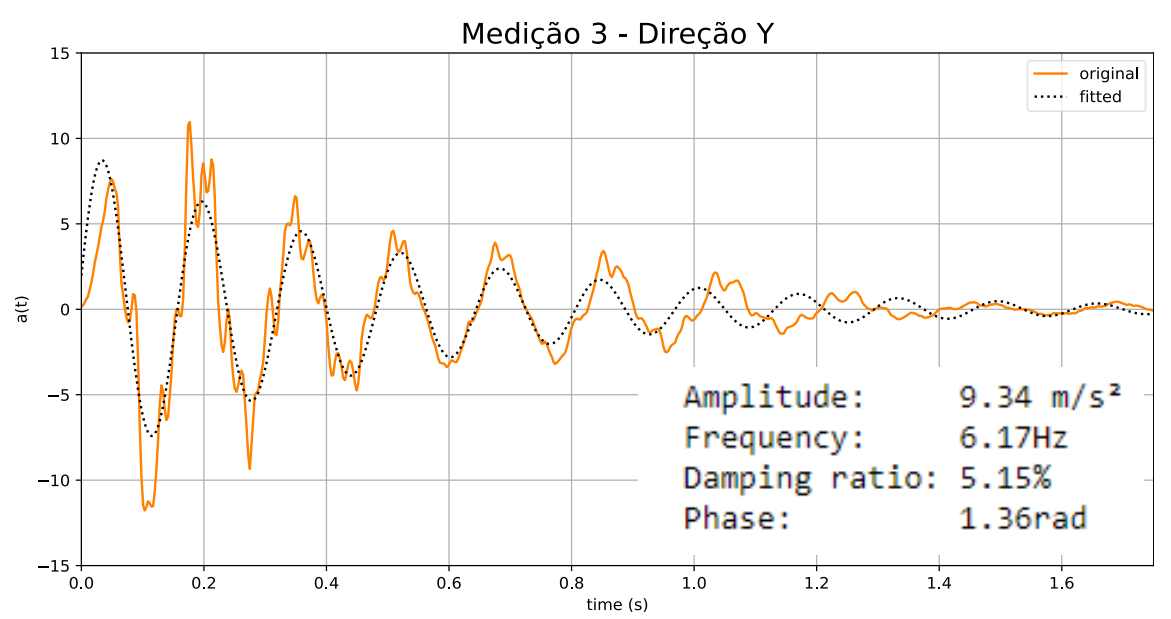

Fonte: Os autores (2022)

Para a obtenção da frequência, foi programada a Transformada Rápida de Fourier (FFT), a partir do sinal de aceleração. Após, para efeito de visualização é plotado o estimador do Espectro de Potência do sinal, i.e., o Periodograma, onde é possível analisar a frequência fundamental e as outras frequências possivelmente excitadas, conforme as Figuras 11, 12, 13 relativas a direção X, e as Figuras 14, 15 e 16 relativas a direção Y.

Figura 11 - Medição 1: frequência na direção X

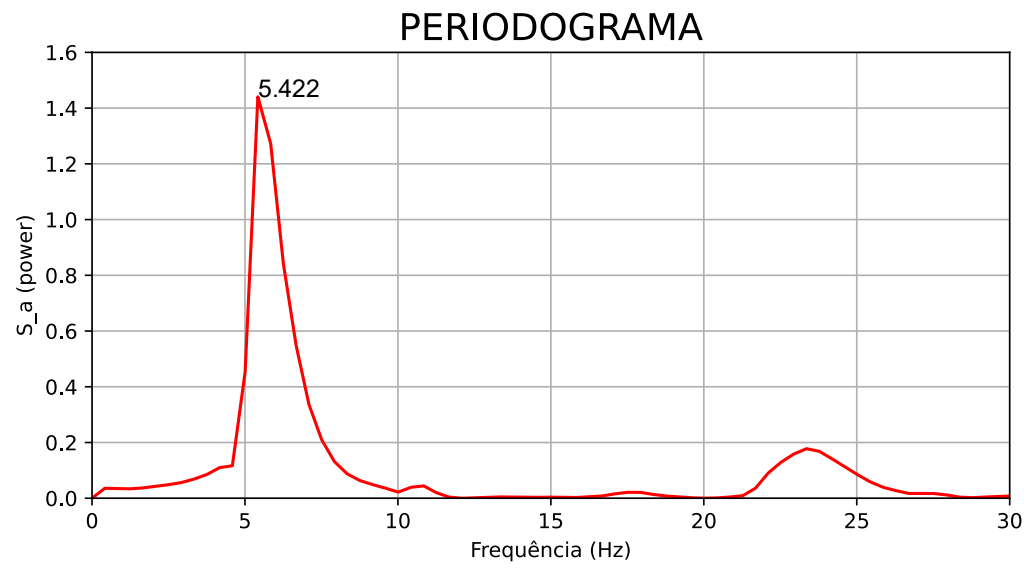

Fonte: Os autores (2022) 
Figura 12 - Medição 2: frequência na direção X

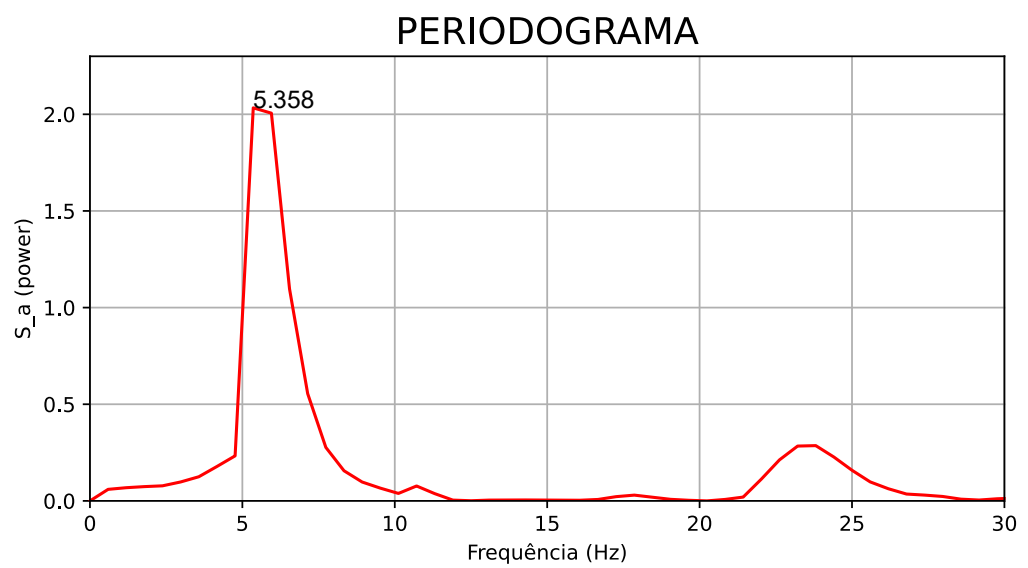

Fonte: Os autores (2022)

Figura 13 - Medição 3: frequência na direção X

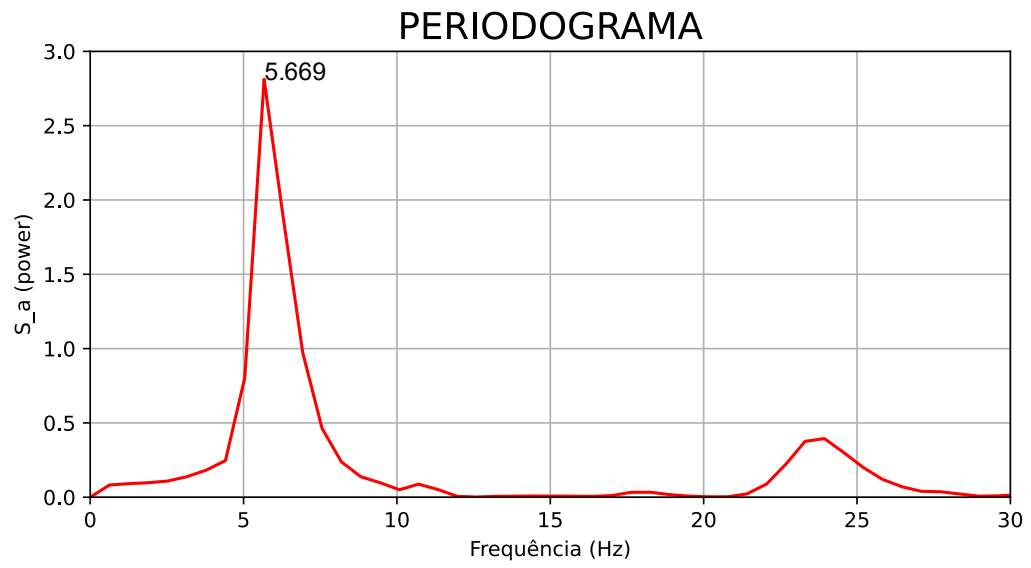

Fonte: Os autores (2022)

Figura 14 - Medição 1: frequência na direção Y

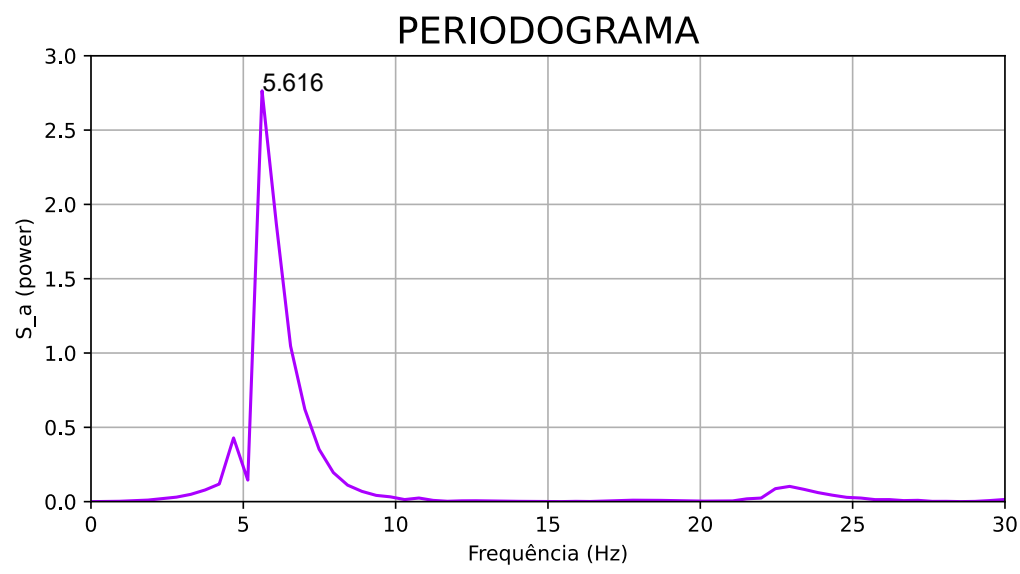

Fonte: Os autores (2022) 
Figura 15 - Medição 2: frequência na direção Y

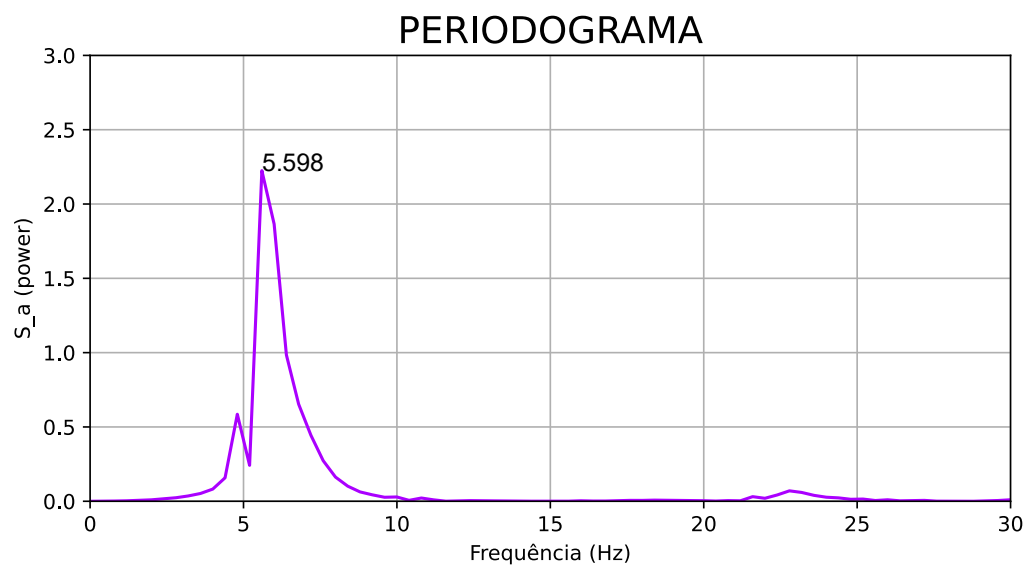

Fonte: Os autores (2022)

Figura 16 - Medição 3: frequência na direção Y

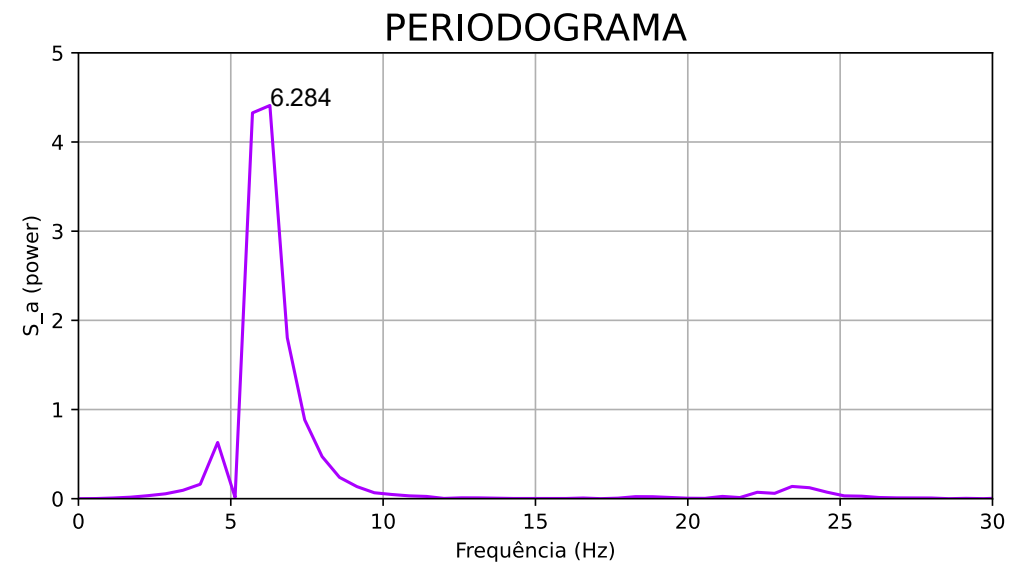

Fonte: Os autores (2022)

\section{MODELO ANALÍTICO}

De acordo com Chopra (2017), para a resolução da análise modal em estruturas com múltiplos graus de liberdade (MDOF), é possível obter uma resposta com nível razoável de precisão através da condensação estática, i.e., limitação dos graus de liberdade, em que diminui consideravelmente o custo computacional, simplificando a resolução do problema.

O modelo analítico para a resolução da análise modal do modelo reduzido CAARC foi o Shear Building, em que considera a viga/laje como elementos infinitamente rígidos e com massas concentradas, resultando em graus de liberdade apenas a translação devido a flexão dos pilares. Cada pavimento possui um grau de liberdade, conforme pode ser observado na matriz de rigidez e de massa apresentada nas Equações 3 e 4 a seguir, em que a estrutura é simplificada a 5 graus de liberdade, devido aos 5 pavimentos. 
$\mathrm{K}=\left[\begin{array}{ccccc}2 k & -2 k & 0 & 0 & 0 \\ -2 k & 4 k & -2 k & 0 & 0 \\ 0 & -2 k & 4 k & -2 k & 0 \\ 0 & 0 & -2 k & 4 k & -2 k \\ 0 & 0 & 0 & -2 k & 4 k\end{array}\right]$

$\mathrm{M}=\left[\begin{array}{ccccc}m & 0 & 0 & 0 & 0 \\ 0 & m & 0 & 0 & 0 \\ 0 & 0 & m & 0 & 0 \\ 0 & 0 & 0 & m & 0 \\ 0 & 0 & 0 & 0 & m\end{array}\right]$

Em que $k=\frac{12 E I}{L^{3}}$ é a rigidez a flexão do pilar engastado perfeitamente, e $m$ a massa do pavimento (laje + viga) representada aravés da placa de acrílico.

A avaliação das incertezas bem como a propagação de erro relacionadas ao ensaio experimental foram implementadas no modelo analítico através da análise de Monte Carlo utilizando 10.000 iterações no cálculo da análise modal. O script encontra-se no mesmo endereço do GitHub mencionado anteriormente através do arquivo ModalAnalise.ipynb. A apresentação dos dados estatísticos referente ao modelo reduzido é apresentada na Tabela 4.

Tabela 4 - Dados estatísticos do modelo

\begin{tabular}{ccccc}
\hline & Elemento & Valor & Distribuição & $\begin{array}{c}\text { Coef. de } \\
\text { Variação [CV] }\end{array}$ \\
\hline Inércia $\left[\mathrm{m}^{4}\right]$ & Pilar & $9.54 \mathrm{E}-13$ & Normal & $1 \%$ \\
\hline Massa $[\mathrm{kg}]$ & Laje/viga & 0.1215 & Normal & $1 \%$ \\
\hline Altura [m] & Pilar & 0.09 & Normal & $0.5 \%$ \\
\hline Mód. Elast. [MPa] & Pilar & 200 & \multicolumn{2}{c}{ Determinístico } \\
\hline
\end{tabular}

Fonte: Os Autores (2022)

\section{MODELO NUMÉRICO}

Para obtenção de uma análise comparativa mais apurada e avaliação do modelo reduzido experimental, elaborou-se um modelo numérico tridimensional através do 
software de elementos finitos Abaqus/CAE de forma a resolver problema de análise modal e identificar a frequência fundamental da estrutura bem como os principais modos de vibração.

A estrutura inteira foi discretizada com o mesmo tamanho de malha correspondente a $0.01 \mathrm{~mm}$, e as ligações entre os elementos laje/viga e os pilares foram definidas com restrição tipo tie, i.e., conexão rígida entre os elementos de forma a retratar o comportamento monolítico.

A componente laje/viga foi modelada em um elemento sólido hexaédrico com 8 nós e integração reduzida. O pilar foi modelado em um elemento sólido tetraédrico com 10 nós e sem integração reduzida. Ambos os elementos estão presentes na biblioteca fornecida pelo software, e podem ser consultados através da nomenclatura C3D8R e C3D10, respectivamente (ABAQUS, 2014).

As propriedades dos materiais de cada componente podem ser verificadas na Tabela 5 a seguir.

Tabela 5 - Propriedade dos materiais do modelo numérico

\begin{tabular}{ccc}
\hline Elemento & Pilar & Laje/Viga \\
\hline Material & Aço SAE 1010 & Acrílico \\
\hline Mód. Elasticidade E $[\mathrm{MPa}]$ & 200 & 3.28 \\
\hline Massa específica $\rho\left[\mathrm{kg} / \mathrm{m}^{3}\right]$ & 8340.70 & $4389.14^{*}$ \\
\hline Coef. Poisson v & 0.3 & 0.37 \\
\hline
\end{tabular}

* massa específica equivalente considerando a chumbada proporcional.

Fonte: Os Autores (2022)

\section{RESULTADOS E DISCUSSÃO}

\section{Avaliação do modelo experimental}

A média da frequência fundamental da estrutura do modelo reduzido após as 3 medições na direção X e 3 medições na direção Y, tal como o desvio padrão é apresentado na Tabela 6 que se segue. É possível verificar que as frequências medidas foram bem próximas conforme o protótipo CAARC estabelecido. 
Tabela 6 - Frequência fundamental do modelo experimental

\begin{tabular}{ccc}
\hline Frequência $[\mathrm{Hz}]$ & Média $[\mathrm{Hz}]$ & Desvio Padrão $[\mathrm{Hz}]$ \\
\hline Direção X & 5.56 & 0.12 \\
\hline Direção Y & 5.87 & 0.25 \\
\hline
\end{tabular}

Fonte: Os Autores (2022)

A frequência fundamental média na direção $\mathrm{Y}$ foi de ordem pouco superior comparado a direção X, motivo este possível causado pela influência dos cabos que ligam o acelerômetro ao Arduino, resultando em um enrijecimento da estrutura quando movimentada.

Não obstante, a terceira grandeza de base, i.e., a escala de frequência $\lambda_{f}$ pode ser então determinada através da Equação 5 que considera a menor frequência fundamental média (direção X) para efeito de cálculo:

$$
\lambda_{f}=\frac{f_{m}}{f_{p}}=\frac{5.56}{0.2}
$$

Em que $f_{m}$ é a frequência do modelo reduzido; $f_{p}$ a frequência do protótipo;

Com as três grandezas de base definidas é possível calcular quaisquer grandezas derivadas de interesse, e.g., a escala de velocidade básica do vento a ser simulada em um possível túnel de vento. A Tabela 7 apresenta algumas das grandezas derivadas calculadas:

Tabela 7 - Grandezas derivadas

\begin{tabular}{ccc}
\hline Grandeza & Escala \\
\hline Aceleração & $1 / \lambda_{a}$ & 0.51 \\
\hline Velocidade & $1 / \lambda_{v}$ & 14.34 \\
\hline Rigidez a flexão & $1 / \lambda_{E I}$ & $5.61 \mathrm{E} 12$ \\
\hline
\end{tabular}

Fonte: Os Autores (2022)

Portanto, considerando um cenário de velocidade básica do vento na região sudeste do Brasil, de acordo com a NBR 6123/88, por meio do mapa de isopletas, a velocidade de $45 \mathrm{~m} / \mathrm{s}$ seria escalada a uma velocidade correspondente aproximada de $3.139 \mathrm{~m} / \mathrm{s}$ no túnel de vento. 


\section{Avaliação do modelo analítico}

O resultado da análise modal com a consideração de incertezas e propagação de erro calculada para o modelo analítico Shear Building através do método de Monte Carlo após 10.000 iterações é exibido na Figura 17 e na Figura 18. Por conseguinte, o valor médio da frequência fundamental da estrutura é apresentado na Tabela 8 seguidamente.

Figura 17 - Função de densidade e probabilidade (PDF)

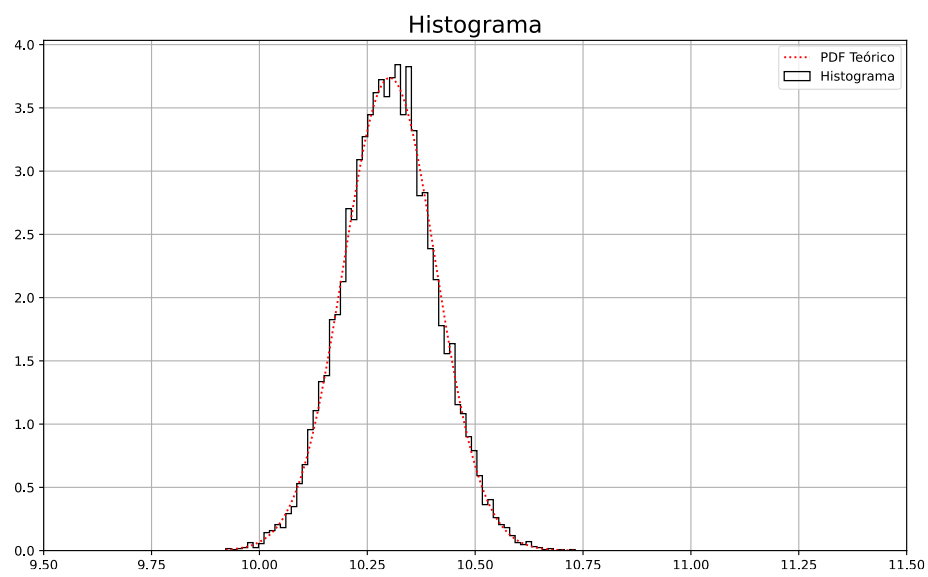

Fonte: Os autores (2022)

Figura 18 - Função de probabilidade acumulada

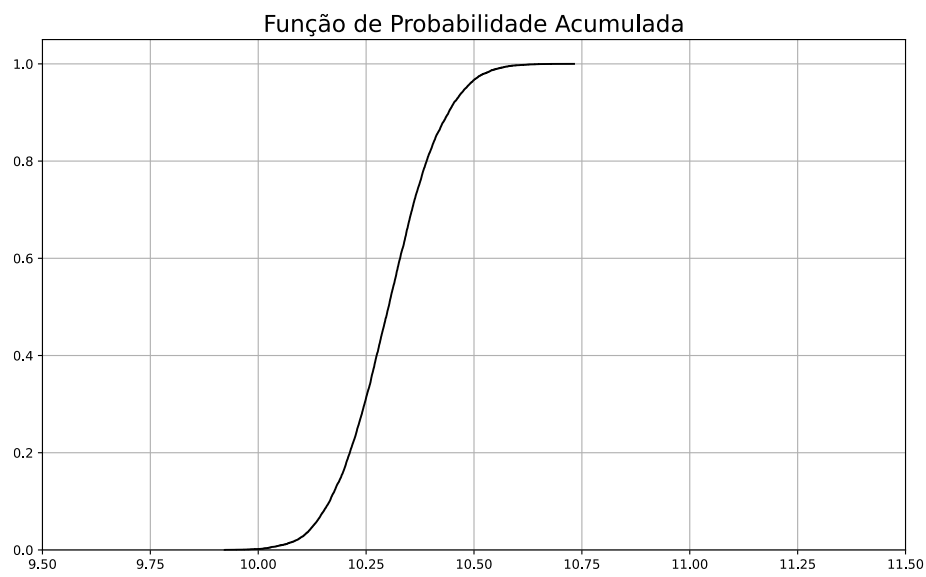

Fonte: Os autores (2022)

Tabela 8 - Frequência fundamental do modelo analítico

\begin{tabular}{ccc}
\hline Média $[\mathrm{Hz}]$ & Desvio Padrão $[\mathrm{Hz}]$ & $\begin{array}{l}\text { Coeficiente de } \\
\text { variação }[\mathrm{CV}]\end{array}$ \\
\hline 10.30 & 0.11 & $1.04 \%$ \\
\hline
\end{tabular}

Fonte: Os Autores (2022)

\section{Avaliação do modelo numérico}


A frequência fundamental do modelo numérico calculada após a análise modal tridimensional foi de $8.035 \mathrm{~Hz}$. Os principais modos de vibração da estrutura podem ser conferidos na Figura 19, sequencialmente:

Figura 19 - Modos de vibração
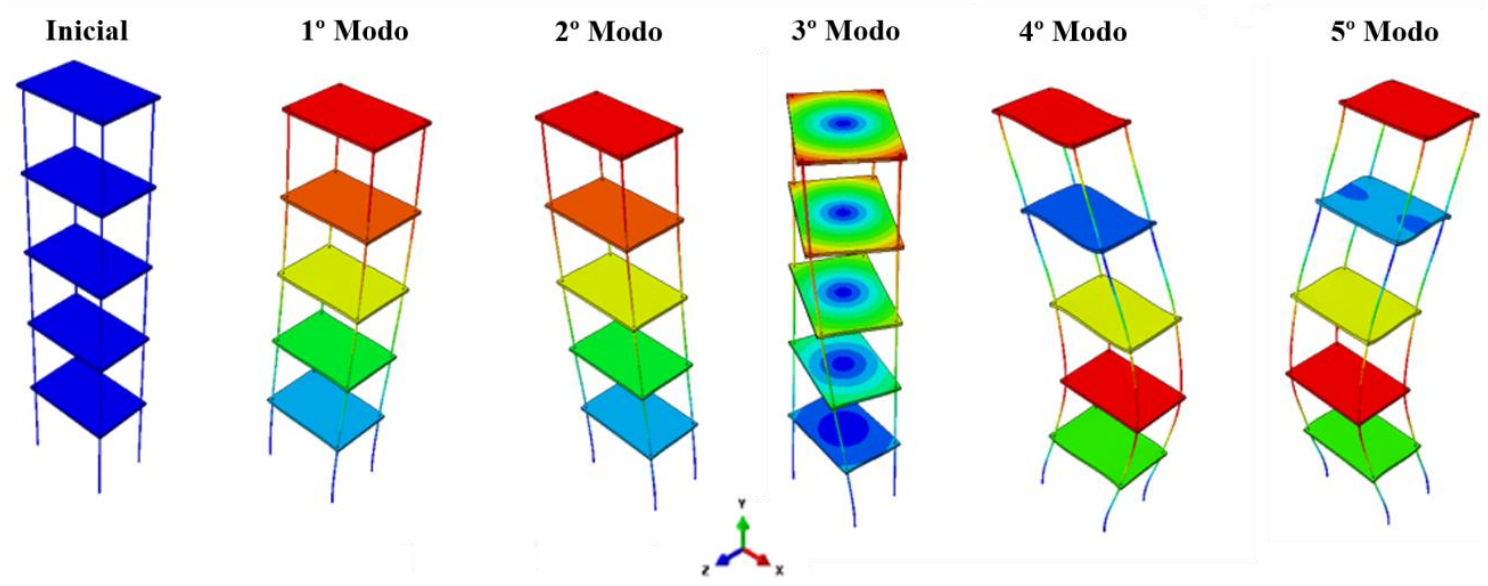

Fonte: Os Autores (2022)

\section{Análise comparativa entre os três modelos}

A comparação entre os modelos reduzidos compreende exclusivamente na frequência relativa ao primeiro modo de vibração da estrutura, pois diferentemente do modelo analítico e numérico, o ensaio experimental realizado foi capaz de medir apenas a frequência fundamental. A Tabela 9 ressalta as diferenças encontradas entre cada modelo.

Tabela 9 - Comparação entre as frequências fundamentais dos modelos

\begin{tabular}{cccc}
\hline Modelos Reduzidos: & Experimental & Analítico & Numérico \\
\hline Freq. Fundamental $[\mathrm{Hz}]$ & 5.56 & 10.30 & 8.04 \\
\hline
\end{tabular}

Fonte: Os Autores (2022)

A consideração da idealização do comportamento de engastamento perfeito entre os elementos estruturais é retratada tanto no modelo analítico quanto no numérico. $\mathrm{O}$ primeiro é manifestado através da rigidez $12 \mathrm{EI} / \mathrm{L}^{3}$, ao passo que no segundo é representado através da restrição tipo tie. Deste modo, a razão pela qual a frequência fundamental do modelo experimental foi consideravelmente inferior aos outros pode ser evidenciada devido a dificuldade de garantia desse comportamento de engastamento perfeito entre os elementos estruturais representados pelo fio de arame galvanizado (pilar) 
e pela placa de acrílico com as chumbadas (laje/viga), resultando em uma estrutura mais flexível, i.e., com uma frequência fundamental menor.

Em contrapartida, é notório observar que no modelo Shear Building a rigidez da estrutura é superestimada, pois não é levado em consideração a massa dos pilares, resultando em uma frequência superior ao modelo numérico.

\section{CONCLUSÕES}

Diante do exposto, o trabalho se propôs em demonstrar a importância da realização de uma análise experimental juntamente com a análise teórico e numérica do modelo reduzido CAARC, ressaltando as diferenças encontradas entre cada modelo. Os pontos as serem destacados são: a escala de aceleração obtida como grandeza derivada não foi 1:1, o que limita a utilização do modelo apenas para análises lineares; a escala de velocidade obtida como grandeza derivada condiz com escalas utilizadas usualmente em túneis de vento; a construção de modelo reduzido capaz de simular bem o comportamento idealizado ou do protótipo não é uma tarefa fácil de ser realizada e deve ser feita com análise de propagação de erro; o amortecimento calculado em todas as medições realizadas foi superior a 1\%; o acelerômetro MPU-6050 em conjunto com o Arduino foi eficiente na medição da frequência fundamental de vibração da estrutura.

Por fim, dentre as abordagens, o modelo experimental foi o mais flexível por não conseguir garantir a rigidez de engastamento perfeito entre os elementos; o modelo analítico o mais rígido, por não considerar a influência da massa dos pilares; e o modelo numérico mostrou ser o mais coerente com modelo experimental.

\section{REFERÊNCIAS}

ABAQUS. Online Documentation, Version 6.14, Dassault Sistèmes. France, 2014. ASSOCIAÇÃO BRASILEIRA DE NORMAS TÉCNICAS. ABNT NBR 6123: Forças devidas ao vento em edificações, Rio de Janeiro.

BALENDRA, T. Vibration of Building to Wind and Earthquake Loads. SpringerVerlag, 1993.

BECK, A. T. Confiabilidade e segurança das estruturas. Elsevier Brasil, 2019.

CARNEIRO, F. L. Análise Dimensional e Teoria da Semelhança e dos Modelos

Físicos. $2^{\text {a }}$ ed. Rio de Janeiro: Editora UFRJ, 1993, 256p. 
CHOPRA, A. K. Dynamics of structures. theory and applications to. Earthquake Engineering, 2017.

MELBOURNE, W.H. Comparison of measurements on the CAARC standard tall building model in simulated wind flows. Journal of Wind Engineering and Industrial Aerodynamics, Amsterdam, v. 6, p. 73-88, 1980.

OLIVEIRA, M. G. K. Desenvolvimento de uma balança dinâmica de três graus de liberdade para estudo dos efeitos de flexo torção em edifícios altos submetidos à ação do vento. Tese de Doutorado. Programa de Pós Graduação em Engenharia Civil. Universidade Federal do Rio Grande do Sul. Porto Alegre, 2009. 207 f.

ROCHA, M. M. Introdução a Teoria de Vibrações - Notas de Aula de PEC0025. Notas de aula, Departamento de Engenharia Civil, Universidade Federal do Rio Grande do Sul, Porto Alegre, 2021.

ROSSATO, B. B. Otimização de parâmetros de amortecedores de massa sintonizados para uso em edifícios altos submetidos à ação do vento. Dissertação de Mestrado. Programa de Pós-Graduação em Engenharia Civil, Universidade Federal do Rio Grande do Sul, Porto Alegre, 2020. 116 f.

ROSSATO, L. V. Otimização de amortecedores de massa sintonizados em estruturas submetidas a um processo estacionário. Dissertação de Mestrado. Programa de Pós-Graduação em Engenharia Mecânica, Universidade Federal do Rio Grande do Sul, Porto Alegre, 2017. 118 f.

SARTORI, F. C. Uma comparação de métodos para avaliar a resposta dinâmica de edifícios altos em túnel de vento . Dissertação de Mestrado. Programa de Pós Graduação em Engenharia Civil. Universidade Federal do Rio Grande do Sul. Porto Alegre, 2010. $168 \mathrm{f}$.

VANIN, D. D. Resposta dinâmica de edifícios altos frente à ação do vento: comparação de técnicas experimentais em túnel de vento . Dissertação de Mestrado. Programa de Pós Graduação em Engenharia Civil. Universidade Federal do Rio Grande do Sul. Porto Alegre, 2011. 122 f. 